\title{
Efficacy and Adverse Effects of Dental Bleaching in the Office: Literature Review
}

\author{
Bruna Naiara Molina ${ }^{1}$, Luis Eduardo Genaro ${ }^{2 *}$, Maria Carolina Stoco Fazanaro ${ }^{1,2}$, Gabriela \\ Ohata $^{1}$ and Andréa Abi Rached Dantas ${ }^{1}$ \\ ${ }^{1}$ Department of Restorative Dentistry, Araraquara Dental School, São Paulo State University (UNESP), Brazil \\ ${ }^{2}$ Department of Morpholog, Genetics, Orthodontic and Pediatric Dentistry, São Paulo State University (UNESP), Brazil
}

*Corresponding author: Luis Eduardo Genaro, São Paulo State University (UNESP), School of Dentistry, Department of Morpholog, Genetics, Orthodontic and Pediatric Dentistry, Araraquara, São Paulo, Brazil

\begin{tabular}{|c|}
\hline ARTICLE INFO \\
\hline Received: 亚 April 19, 2021 \\
\hline Published: 慧 April 28, 2021 \\
\hline $\begin{array}{l}\text { Citation: Bruna Naiara M, Luis Eduardo } \\
\text { G, Maria Carolina Stoco F, Gabriela O, } \\
\text { Andréa Abi Rached D. Efficacy and Adverse } \\
\text { Effects of Dental Bleaching in the Office: } \\
\text { Literature Review. Biomed J Sci \& Tech Res } \\
\text { 35(3)-2021. BJSTR. MS.ID.005693. }\end{array}$ \\
\hline
\end{tabular}

Keywords: Tooth bleaching; Hydrogen peroxide; Carbamide peroxide

\begin{abstract}
Background: Perform a literature review on dental bleaching in order to evaluate its effectiveness and its adverse effects.

Materials and Method: For this literature review, electronic searches were performed for articles related to the keywords: "Dental bleaching", "Hydrogen peroxide", "Carbamide peroxide", in the databases Pubmed, Lilacs and Scielo, among the years 2009 and 2019. The articles were cited in chronological order from the year 2009.
\end{abstract}

Result: After searching the articles, 25 studies were selected that analyzed the effectiveness of tooth whitening and its effects.

Conclusion: The association of office and home techniques can minimize possible damage to dental structures. The professional, to obtain favorable results in the use of the office whitening technique, must know the product he will use, follow the manufacturer's recommendations for the application and guidance to the patient, respect the soft tissue protection technique, use products that minimize sensitivity, as well as individualizing each treatment.

Clinical Significance: The literature reports that tooth whitening is a technique that is safe, efficient and has no harmful effects on dental enamel when an appropriate and individualized protocol is performed for each patient.

\section{Introduction}

Tooth whitening can be considered as one of the simplest, quickest and minimally invasive treatments for the aesthetic improvement of darkened teeth, in addition to presenting a low cost when compared to other restorative aesthetic alternatives $[1,2]$. Thus, it has become much sought after by patients in search of a better smile appearance. The change in the color of the teeth can occur due to intrinsic and extrinsic factors. Intrinsic factors can result from dental trauma, mistaken endodontic treatments, intrapulpal hemorrhage, pulp necrosis and certain dental diseases or defects. Extrinsic factors, on the other hand, result from the accumulation of chromophoric substances resulting from foods and beverages such as coffee, red wines, teas and spices, the use of tobacco and some medicines [3-5]. There is a treatment protocol for each change. Therefore, the correct diagnosis of the causal agent is essential in order to establish the best treatment plan and thus obtain the appropriate prognosis [6]. Among the different whitening techniques, we have homemade tooth whitening and office dental whitening. We can also have an association between both techniques. Homemade tooth whitening consists of the patient applying the whitening gel in personalized trays for a certain time, under the supervision of the dentist. It stands out for the use of low concentration bleaching agents $[3,4,7]$.

Dental whitening in the office consists of applying gels based on carbamide peroxide above $20 \%$ and hydrogen peroxide above 
$10 \%$ in the dental office. This technique has the advantage of being directly controlled and supervised by the professional, so that there is no contact with soft tissues and gel ingestion, does not depend on the collaboration of the patient and exhibits immediate results, and can be performed in just one clinical session, $[3,4,8]$ therefore, it is indicated for patients who want fast, effective and stable results and who prefer not to use the home whitening tray.7,9 For the office dental whitening procedure, the soft tissues are protected prior to application, so that no gingival inflammation, burns and ulcerations in the soft tissues occur and, only after this step, the whitening gel is applied on the teeth surface [2,9-11]. Tooth whitening occurs when whitening agents penetrate enamel and dentin, releasing free oxygen radicals that break the double bonds present in the organic compounds responsible for the color change and transform the pigment molecules into smaller, less complex and, consequently, more clear $[1,3,6,7,10,12]$. In the dental office the most used bleaching agent is hydrogen peroxide $35 \%$, as it has low molecular weight and has a high penetrating power in enamel and dentin [10].

To speed up the whitening process in the dental office, light sources such as halogen lamps, LEDs, plasma arcs and lasers can be used, which act by activating the bleaching agents by increasing their temperature and causing the release of free radicals and improving treatment effectiveness [2-4,8,13,14]. However, this information has generated controversy, as some authors demonstrate that the effectiveness of these light sources in assisting the whitening treatment is still inconclusive and that the use of these does not alter the final result $[2,6,8,13]$. In addition, there are studies showing that the heat released by these sources may be in excess of the tolerable limit for the dental pulp, causing pulp inflammation and contributing to the increase in tooth sensitivity $[6-8,10,13]$.

The effectiveness of office dental whitening has been verified by numerous studies, $[11,15]$ the high concentration of bleaching gels used in the office results in faster and more efficient bleaching [12]. Although there are protocols that reduce the contact time between the bleaching agent and the tooth structure, there are several adverse effects found in the literature, the most common being tooth sensitivity $[4,9,11,12,16-212]$, which is generally associated with the ability of hydrogen peroxide to diffuse rapidly through enamel and dentin and penetrate the pulp, causing intense damage $[3,9,17,19,20]$, as pulp inflammatory reactions $[11,19,21]$, reduced cell proliferation and reduced pulp repairing capacity, which can lead to reversible pulpitis or even tissue necrosis $[3,14,22]$. Morphological changes in enamel and dentin and changes in mineral content can also occur resulting in the presence of erosions, porosities (which can lead to possible bacterial colonization), 23 decreased microhardness and nano hardness, increased surface roughness and dental demineralization, as well as fracture toughness $[4,5,12,15-17,21,23]$.
The side effects of office bleaching may be associated with the composition of bleaching agents, concentration, since the higher the concentration, the greater the probability of hydrogen molecules reaching the dentinal tubules, the $\mathrm{pH}$ values (the lower $\mathrm{pH}$ may be the main reason for the negative effects) the treatment time, the increase in the temperature of the pulp with the use of light and the different application techniques [5,11,14-16,19-21,23]. Thus, it is necessary to take measures to minimize the possible adverse effects during the bleaching treatment [15]. The use of laser therapy can minimize tooth hypersensitivity by stimulating cell differentiation, increasing tissue repair and reducing inflammation.3 Calcium and / or fluoride can also be added to the composition of bleaching gels and the application of fluoride after treatment to reduce the risk of demineralization or loss of mineral enamel content $[16,17,202]$. Another alternative would be to decrease the concentration of bleaching gels used in the office, generating less cytotoxicity and damage to periodontal tissue.9 In addition, it is better to opt for the use of bleaching gels with neutral or alkaline $\mathrm{pH}$, as they have lower risks and intensity of tooth sensitivity than those with acid $\mathrm{pH}[15,19$,$] . Thus, a literature review that shows us the efficacy and$ adverse effects of dental bleaching in the office becomes relevant and important.

\section{Objective}

The objective of this study was to perform a literature review on dental bleaching in the office, consulting articles in the Pubmed, Lilacs and Scielo databases, between the years 2009 and 2019, to assess their effectiveness, advantages, disadvantages and adverse effects.

\section{Material and Method}

For this Literature Review, electronic searches were performed for articles related to the keywords: "Dental bleaching", "Hydrogen peroxide", "Carbamide peroxide", in the databases PubMed, Lilacs and Scielo, between the years 2009 and 2019.

\section{Literature Review}

25 articles were included after searching the database, these studies are described below based on some categories.

\section{Use of Light Sources}

Nunes et al. [2] evaluated the effect of using halogen lamps in vivo as catalysts for a gel based on hydrogen peroxide at $37.5 \%$, used for tooth whitening in the dental office. For this, thirteen patients underwent tooth whitening with $37.5 \%$ hydrogen peroxide (Whiteness HP Maxx, FGM / Joinvile, SC, Brazil), who had their upper arch divided and, in each hemiarch, was performed the bleaching treatment with hydrogen peroxide was randomly performed, with one hemiarch being associated with halogen lamp lighting and the other hemiarch was not used any light source. 
After the analysis performed by the t-student test, it was found that there was no significant difference in bleaching with the techniques performed with and without the presence of light. Thus, it was concluded that in the dental whitening technique performed in the office it is not necessary to use halogen lamps, because although it does not affect the effectiveness of hydrogen peroxide, it also did not improve its action.

Maia and Catão [1] evaluated the effectiveness of tooth whitening with $35 \%$ hydrogen peroxide activated by laser and LED light sources, in artificially darkened bovine teeth. After staining the teeth were divided into two groups, with group 1 using $35 \%$ hydrogen peroxide activated by LED and group 2 using 35\% hydrogen peroxide activated by laser. From the results obtained it was found that the two techniques achieved a high level of whitening, however the technique that used the Laser to activate $35 \%$ hydrogen peroxide showed superior results when compared to the technique that used the LED. Maran et al. [8] performed a systematic literature review comparing the teeth whitening performed in the dental office with and without the use of light sources, evaluating the effectiveness and sensitivity of the teeth. For this, twenty-one randomized clinical trials were studied. The concentration of hydrogen peroxide varied between 15\% and $38 \%$ and the application protocol for tooth whitening in the dental office was variable. Different types of light activation were used with different protocols. Four studies used halogen light, ten used LEDs / lasers, three used only LEDs, five used metal halide light, four used only a laser source, three used a plasma arc lamp and one used a laser. In some studies, the light was applied for the same period of time as the gel and in other studies the light was applied for a few minutes with a specific interval between applications. Theoretically, heat and light sources can accelerate the decomposition of hydrogen peroxide to form free oxygen radicals, increasing the effectiveness of whitening. However, this hypothesis was not proven in the studies studied, because from the results obtained in the study and, if the variations in the different protocols are not considered, it was observed that the effectiveness and the risk of tooth sensitivity of the tooth whitening performed in the office were not influenced by the use of light, regardless of the concentration of hydrogen peroxide.

\section{Acid or Neutral Hydrogen Peroxide?}

Sun et al. [15] evaluated what the effects of $30 \%$ neutral and acid hydrogen peroxide would be on human dental enamel, checking its chemical structure, mechanical properties, surface morphology and tooth staining. In the experiment, 27 pre-molars were selected, randomly divided into 3 experimental groups: acid group, where the samples were immersed in $4 \mathrm{~mL}$ of $30 \%$ hydrogen peroxide solution and $\mathrm{pH} 3.6$; neutral group, where the samples were immersed in a mixture of $4 \mathrm{ml}$ of $30 \%$ hydrogen peroxide solution and $\mathrm{pH} 7.0$ and $5 \mathrm{ml}$ of saturated $\mathrm{NaOH}$ solution; and the control group, where the samples were immersed in $4 \mathrm{~mL}$ of distilled water with $\mathrm{pH}$ 6.8. ATR-FTIR spectroscopic analysis, Raman spectroscopy, atomic force microscopy (AFM), microhardness test and color measurements were performed before and after treatments to perform the evaluations. After the analysis, it was concluded that major changes were found in the acid group after treatment, while small changes were detected in the neutral and control groups. Thus, it was found that hydrogen peroxide at 30\% in neutral concentration had the same efficacy in tooth whitening and caused less deleterious effects on the enamel than hydrogen peroxide at $30 \%$ in acid concentration.

Acuña et al. [19] evaluated the efficacy, the enamel surface morphology after the bleaching treatment and the concentration of hydrogen peroxide in the pulp chamber of teeth bleached with $40 \%$ hydrogen peroxide at different pHs. Forty-five healthy premolars were used for the study. Of these, forty premolars were randomly divided according to the $\mathrm{pH}$ of the bleaching gel: 5.1; 6.3; 7.0 and control (no bleaching, using distilled water on the enamel surface). From the results obtained, it was found that, regardless of the $\mathrm{pH}$ of the bleaching products, all groups presented hydrogen peroxide in the pulp chamber, however the pH 5.1 group showed the highest diffusion of hydrogen peroxide when compared to the bleaching gels. with neutral and alkaline $\mathrm{pH}$. Regarding the color change and the enamel morphology, no significant difference was detected between the groups. Thus, to minimize damage to pulp tissues, neutral and alkaline bleaching gels are suggested.

\section{Lightener with or without Light Source}

Guth et al. [6] studied, through a clinical case report, dental bleaching of vital teeth in the office using hydrogen peroxide gels Whiteness HP Blue 20\% and Whiteness HP Maxx 35\% without using a light source. According to the results obtained, it was found that the office whitening treatment was effective with the two products used and the degree of sensitivity was similar in both hemiarchs. Mondelli at al. [20] evaluated the color change, stability and tooth sensitivity resulting from different whitening techniques. For this, 48 patients were selected randomly divided into five groups, which were: group 1, which used 35\% hydrogen peroxide (Lase Peroside - DMC equipment, São Carlos, SP, Brazil) + hybrid light (Laser LED / Diode, Whitening Lase II DMC Equipment, São Carlos, SP, Brazil); group 2, which used 35\% hydrogen peroxide; group 3, which used $38 \%$ hydrogen peroxide (X-traBoost - Ultradent, South Jordan UT, USA) + hybrid light; group 4, which used 38\% hydrogen peroxide; and ; group 5, which used 15\% carbamide peroxide (Opalescence PF - Ultradent, South Jordan UT, USA). For groups 1 and 3, hydrogen peroxide was applied to the enamel surface, activating the gel by hybrid light. For groups 2 and 4, hydrogen peroxide was applied to the enamel surface, without the use of light, in the dental office and for group 5, patients received instructions and 15\% carbamide peroxide was applied for 10 days. at home. Patients underwent the 
EVA questionnaire to measure tooth sensitivity before bleaching. From the results obtained, it was found that the five techniques and whitening gels used were effective in teeth whitening, however the home whitening group (group 5) showed greater whitening compared to the whitening agents used in the dental office. Among the office whitening groups, with or without light activation (groups 1, 2, 3 and 4), there were no significant differences in effectiveness, however the groups in which hybrid light was used for activation demanded less time for whitening. In the evaluation of tooth sensitivity, it was found that statistical differences were observed only immediately after bleaching, where the home bleaching group (5) due to the low concentration of the gel, presented the lowest index of tooth sensitivity, and the bleaching groups in the office $(1,2,3,4)$, experienced higher levels of sensitivity, but after 24 hours, the degree of sensitivity decreased and returned to normal levels, in 7 days, for all groups evaluated. It was not possible to determine differences in the degree of sensitivity between the groups activated or not activated by hybrid light.

\section{Temperature Influenced by Tooth Bleaching}

Loretto et al. [14] evaluated the increase in the temperature of the pulp chamber influenced by three bleaching agents, using the photoactivated tooth whitening technique performed in a dental office. For this, a healthy and extracted human upper central incisor was selected. The remaining pulp that was in the pulp chamber was removed and the root canal was enlarged, allowing the introduction of the thermometer sensor. The pulp chamber was filled with thermal paste, which allowed heat transfer from the dental walls to the thermocouple sensor during the bleaching treatment. To whiten teeth, three photosensitive whitening agents (35\% hydrogen peroxide) were used: Whiteness HP (FGM Produtos Odontológicas, Joinville, SC, Brazil), Whiteness HP Maxx (FGM Produtos Odontológicas, Joinville, SC, Brazil) and Lase Peroxide Sensy (DMC, São Carlos), SP, Brazil); and for the activation of the bleaching gels, a high intensity LED source, Flash Lite 1401 (Discus Dental Inc., Culver City, CA, USA) was used. From the results, it was found that exposure to light raised the temperature in the different groups studied, however the application of bleaching gels potentiated the absorption of the heat emitted by the light source, except in the group in which the Lase Peroxide Sensy (DMC) was used. The different increases in the temperature of the pulp chamber for the different groups studied can be explained by differences in the composition of the bleaching gels.

\section{Associated or Clinical Bleaching?}

Rezende et al. [7] evaluated effectiveness, tooth sensitivity and color recurrence in two different tooth whitening techniques: the technique performed in the dental office and the associated technique, which associates the whitening treatment from the office to the home. For this, 30 patients were selected, randomly divided into two groups: the associated whitening group and the office whitening group. No dental bleaching group in the office was used 35\% hydrogen peroxide (Mix One Supreme, Villevie, Joinville, Santa Catarina, Brazil). For the associated bleaching group, dental bleaching was also performed with 35\% hydrogen peroxide following the same protocol as the dental bleaching group, but from the following day, a home whitening technician started using hydrogen peroxide a 6\% (Mix Day, Villevie, Joinville, Santa Catarina, Brazil). Both treatments have similar tooth sensitivity and intensity. It was concluded that both techniques are effective, generate similar results regarding the intensity of tooth sensitivity, an associated tooth whitening technique generates greater color stability, however the office whitening technique shows immediate results. Thus, we must indicate each technique according to the patient's need and preference.

\section{Structural and Chemical Characteristics of Dental Tissues after Bleaching}

Izquierdo-Barba et al. [23] evaluated the effects of tooth whitening with $38 \%$ hydrogen peroxide in relation to the morphological, chemical and structural characteristics of human enamel and dentin. For that, third molars were obtained that needed to be extracted by orthodontic indication. One half of each crown was subjected to bleaching treatment with 38\% hydrogen peroxide for 20 minutes and the other half was not treated. For analysis of the samples, environmental scanning electron microscopy, electronic probe microanalyzer and X-ray diffraction techniques were used, which allowed the study of the different teeth constituents (enamel and dentin), without the need for prior preparation. From the results obtained, it was found that the structural and chemical characteristics of the enamel and dentin remained unchanged after the whitening treatment, but the enamel morphology was significantly altered, showing channels with very pronounced pores, which lead to greater susceptibility of the enamel to bacterial attack.

Ayres et al. [12] evaluated the increase in dental permeability resulting from bleaching treatment using four high concentration bleaching agents. For this, forty bovine incisors were used, divided into four groups, which had the whitening agents that would be tested: Whiteness HP Blue (FGM Produtos Odontológicas Ltda, Joinville, SC, Brazil) with concentration of hydrogen peroxide at 35\%, Whiteness HP Maxx (FGM Produtos Odontológicas Ltda, Joinville, SC, Brazil), with 35\% hydrogen peroxide concentration, Pola Office Plus (Southern Dental Industries, Bayswater, VIC, Australia) with hydrogen peroxide concentration 37.5\% hydrogen and Opalescence Boost PF (Ultradent Products Inc, South Jordan, UT, USA) with $38 \%$ hydrogen peroxide concentration. The initial permeability of the dental structure was assessed using a permeability equipment and served as a control. It was found that the group that used the whitening agent Whiteness HP Maxx, which 
does not have desensitizing and / or remineralizing agents in its composition, had a greater permeability increase when compared to the groups that used the whitening agent HP Whitening Blue, which contains neutralizing agents and calcium gluconate and the bleaching agent Pola Office Plus, which contains sodium hydroxide added to the composition. The group that used the whitening agent Opalescence Boost showed intermediate values of increased dental permeability, without interfering in the analysis. Thus, it can be concluded that there was an increase in permeability for all groups tested, varying the intensity, according to the product used, and that the absence of desensitizing and / or remineralizing agents can lead to an increase in dental permeability.

Moreira et al. [17] evaluated changes in the content of calcium and phosphorus in dental enamel when subjected to whitening in the dental office using a 35\% hydrogen peroxide solution, with and without calcium, for a long period of time. For this, 10 human teeth with the removed roots were used, divided into two groups, and group 1 used a whitening agent without calcium, the Whiteness of 35\% HPM axx (FGM, Brazil), with which three applications of 15 minutes per session and group 2 that used the whitening agent Whiteness HP Blue 35\% (FGM, Brazil) and hydrogen peroxide $35 \%$ with calcium. A total of 5 sessions were held in both groups. In view of the results obtained by the statistical analysis, it was found that there was no significant change in the amount of calcium and phosphorus after the bleaching treatment and there were no differences between the two groups. Thus, it was concluded that tooth whitening using hydrogen peroxide in high concentrations with or without calcium, does not cause the loss of calcium and phosphorus in the dental enamel.

Pinto et al. [16] evaluated the content of calcium (Ca) and phosphorus (P) present in the enamel after bleaching with high and low concentrations of hydrogen peroxide using full fluorescence of reflection by X-rays and colorimetric spectrophotometry. In this study, 48 human third molars were used, these teeth were distributed in six groups to receive bleaching treatments, and four groups were made up of high concentration whitening agents (for office use): Whiteness HP Maxx / FGM (35\% of hydrogen peroxide); Whiteness HP Blue / FGM (35\% hydrogen peroxide and 2\% calcium gluconate); Pola Office + / SDI (37.5\% hydrogen peroxide and $5 \%$ potassium nitrate) and Opalescence Boost/Ultra dent (38\% hydrogen peroxide, $1.1 \%$ fluoride ion and $3 \%$ potassium nitrate) ; applied to the enamel in three sessions and two groups with lowconcentration whitening agents (for home use): Pola Day / SDI (9.5\% hydrogen peroxide, and 5\% fluoride); White Class 10\% / FGM (10\% hydrogen peroxide, potassium nitrate, calcium and fluoride). The bleaching agents were applied according to the instructions and application times recommended by the manufacturers. It was found that $\mathrm{Ca}$ and $\mathrm{P}$ were not influenced by the agent. In SPEC, Pola Office + , Opalescence Boost, Pola Day and White Class 10\% there was a decrease in Ca over time; there was a significant decrease in
P over time for Pola Office + and White Class $10 \%$. Thus, changes in the content of calcium and phosphate in the enamel during and after bleaching can occur regardless of the presence of fluorine or calcium in the composition of certain brands of bleaching agents, hydrogen peroxide concentration and bleaching techniques used.

Menezes et al. [25] evaluated the impact of tooth whitening using $35 \%$ hydrogen peroxide in relation to the color and translucency of dentin and enamel. For the study, five healthy human molars were selected, and their crowns were sectioned into four slices parallel to the buccal surface. Tooth bleaching was performed with $35 \%$ hydrogen peroxide in the samples, with three applications of 15 minutes each. Afterwards, the samples were stored in water for 7 days, the color measurements were repeated on both backgrounds and the color and translucency changes to the enamel and dentin were calculated and analyzed. From the results obtained, it can be seen that tooth whitening increased the luminosity measured in the enamel and made it a little less translucent, since in dentine there were no significant changes.

De Rosa et al. [18] evaluated in vivo what the effects of the hydrogen peroxide agent would be on dental bleaching in the dental office on the surface of the dental enamel using a reflection confocal microscopy. For this, ten healthy volunteers were selected. A 35\% hydrogen peroxide bleaching agent was used, applied in two 20-minute sessions, with an interval of one week between them, following the manufacturer's instructions. Soon after the whitening procedures, the enamel prisms remained unchanged and white circular reflecting bodies were found on the entire surface of the teeth. One week after bleaching, fewer white circular areas were found on the enamel surface. The textural analysis revealed that there is no statistically significant difference between the images for all analyzed parameters. Thus, it was concluded that there are no deleterious effects on enamel with tooth whitening using hydrogen peroxide.

Karaarslan et al. [3] studied biochemical changes and levels of oxidative stress present in dental tissues after different tooth whitening techniques performed in the dental office. For this, 10 people were selected, whose first four premolars needed to be extracted for orthodontic reasons. Thus, totaling 40 teeth for the study. The premolars were divided into 4 groups where three different bleaching methods containing hydrogen peroxide bleaching agents and a control group were performed. In group 1, the dental surfaces of the premolars were covered with a White20 laser whitening gel (Biolase Technology Inc., San Clemente, CA, USA) containing $46 \% \mathrm{H}_{2} \mathrm{O}_{2}$. The activation of the bleaching gel was performed with a diode laser ( $940 \mathrm{~nm}$; Epic 10, Biolase Technology Inc., San Clemente, CA, USA). In group 2, the tooth surfaces were covered with Whiteness HPMaxx (FGM, Joinville, SC, Brazil) containing 35\% $\mathrm{H} 2 \mathrm{O} 2$ and the bleaching gel was activated with the bleaching mode of the halogen photopolymerization unit (Optilux 
501, Kerr, Orange, CA, USA). In group 3 the tooth surfaces were covered with Whiteness HP Maxx (FGM) containing 35\% $\mathrm{H}_{2} \mathrm{O}_{2}$, for 15 minutes. Group 4 was the control group and there was no dental bleaching procedure. 24 hours after the bleaching procedures, the premolars were extracted and separated at the cementation junction. From the results obtained from the analysis, it was concluded that there were no differences between the bleaching groups in both tissues when evaluating cathepsin B and MMPs.

\section{Effect of Different Products and Whitening Techniques}

Roderjan et al. [22] evaluated the efficacy and possible adverse effects on human teeth submitted to different techniques and materials of tooth whitening performed in a dental office. For the study, 17 participants were selected. The patients were randomly divided into four groups, one of which would be the control group, which did not receive tooth whitening and the others would receive whitening treatments. Gels based on 35\% hydrogen peroxide without calcium (SC) and containing calcium (CC) (Whiteness HP Maxx [CF], Whiteness HP Blue 35 [CC], FGM). Dental sensitivity was greater in the groups bleached with $35 \%$ hydrogen peroxide without calcium, than in the group containing calcium and in the control group. Groups SC 3-15 and SC 1-45 exhibited a zone of necrosis in the coronary pulp tissue associated with deposition of tertiary dentin. In the CC 1-45 group, on the other hand, there were small areas of necrosis and a moderate deposition of reactionary dentin. Thus, it was concluded that the gel based on 35\% hydrogen peroxide containing calcium causes less tooth sensitivity and damage to the pulp tissue and may be more suitable for whitening in the office.

Gonçalves et al. [13] evaluated the effectiveness and levels of tooth sensitivity that could occur after the dental whitening treatment performed in the dental office with $20 \%$ and $35 \%$ hydrogen peroxide in adolescent and young patients. For this, 53 patients were selected to carry out a randomized controlled clinical trial, in which they were randomly divided into different groups using the commercial products Whiteness HP - FGM® (35\% hydrogen peroxide); Whiteness HP Blue Calcium - FGM® (35\% hydrogen peroxide); and Whiteness HP Blue Calcium FGM® (20\% hydrogen peroxide) and each group used a different type of whitening gel in two clinical sessions. For dental bleaching procedures, auxiliary light sources were not used. Thus, from the data obtained and analyzed, it was concluded that tooth whitening performed in a dental office is effective for adolescents and young people, as there were significant differences in color after the whitening sessions. There was a transitory appearance of tooth sensitivity and it was found that the addition of calcium to the whitening treatment contributed to the reduction of sensitivity. However, for a more complete assessment of the effects of whitening treatment in adolescents and young people, additional studies are still needed.

\section{Difference in the Concentration of the Bleaching Product}

Rodrigues et al. [5] studied the effects of hydrogen peroxide concentration on hydrophilicity, roughness, morphology and the mechanical and tribological properties of human dental enamel. To that end, five healthy human molars were extracted and prepared. The samples were stained using a tea solution at room temperature and then washed. Afterwards, they were submitted to bleaching treatment sessions with $6 \%, 15 \%$ and $35 \%$ hydrogen peroxide, with consecutive sessions, depending on the peroxide concentration, in order to obtain a similar level of bleaching among all samples. The results showed that the treatment with the three concentrations of hydrogen peroxide improved the color of the teeth, but they also changed the properties of the enamel. Thus, there was an increase in the roughness of the enamel surface, since the initial average roughness of the teeth was $36 \mathrm{~nm}$ and after treatment, it increased to values between 58 and $162 \mathrm{~nm}$, with the solution with the concentration at $15 \%$ being the least harmful (this is important to verify, as an increase in roughness can increase the retention of biofilm on the dental surface). There was a small decrease in the enamel hardness and an insignificant change in the hydrophilicity of the enamel surface at the three concentrations. There was an increase in the friction and wear coefficients, however, this increase was not directly proportional to the peroxide concentration, as the samples submitted to treatment with the $15 \%$ solution had the least wear and the largest, was with the $35 \%$ solution. From these results, it can be concluded that the $15 \%$ tooth whitening solution produces the least changes in the enamel properties after the whitening treatment when compared to the $6 \%$ and $35 \%$ solutions.

Grazioli et al. [21] evaluated the effectiveness of tooth whitening and changes in enamel surface properties due to different concentrations of hydrogen peroxide. For the study, bovine incisors were obtained. The samples were immersed in a standardized coffee solution. The samples were randomly distributed in four groups to perform the whitening procedure, which one group served as a control and did not undergo the whitening treatment, however the samples were irradiated with a laser light source (Whitening Lase II; DMC Equipment's, São Carlos, SP, Brazil); and three groups used different concentrations of hydrogen peroxide: LP15\% (Lase Peroxide 15\%), LP25\% (Lase Sensy 25\%) and LP35\% (Lase Sensy 35\%), all DMC products. The tooth whitening was performed according to the manufacturers' instructions and was irradiated with the laser light source. The results showed that all gels produced similar efficacy in whitening and only the control group exhibited less color change when compared to the whitened groups. The initial and final pH values of each bleaching gel showed that the higher the percentage of the gel, the lower the $\mathrm{pH}$ measured at the end of the treatment. Regarding the hardness, it was found that the samples cleared with the $15 \%$ gels did not reduce the hardness, presenting the surface similar to the samples 
of the control group. Gels with concentrations at 25\% and 35\% significantly reduced the hardness of the samples and also produced a morphological change in the enamel, with exposure of the prism rods. Thus, it was concluded that gels with a low hydrogen peroxide concentration can be used effectively and safely.

Lima et al. [9] compared, from a randomized double-blind clinical trial, what the cytokine levels, tooth sensitivity and whitening efficacy would be after the application of whitening treatments performed with $15 \%$ and $35 \%$ hydrogen peroxide in the dental office. For the evaluation, 25 volunteers with anterior teeth were randomly selected, who received the whitening treatment in three 15-minute sessions, with an interval of one week per session. To score the dental sensitivity by the participants, a visual analog scale and a verbal numeric scale were used. From the results, it was concluded that the tooth sensitivity was greater when using hydrogen peroxide at $35 \%$ than at $15 \%$. Regarding efficacy, the use of 35\% hydrogen peroxide achieved greater clarification one month after the procedure, compared to $15 \%$ hydrogen peroxide in the same period. The cytokine levels collected from the gingival fluid were similar at both concentrations and indicated the absence of any persistent anti-inflammatory response. Thus, it is understood that the bleaching treatment with $35 \%$ hydrogen peroxide is more effective, but it has greater risks of presenting tooth sensitivity when compared to the bleaching treatment with $15 \%$ hydrogen peroxide.

\section{Effectiveness and Sensitivity after Bleaching Treatment}

Martins et al. [24] compared the effectiveness and sensitivity of teeth that could occur as a result of tooth whitening performed in the dental office in two different techniques: one technique with a single application performed in 40 minutes and another, with two applications of 20 minutes. The 38\% hydrogen peroxide bleaching agent was used. For this, forty-four patients participated in the study, the bleaching agent was applied in a single application of 40 minutes and on the other side were two applications of 20 minutes, according to the manufacturer's instructions, in two sessions, with an interval of one week between them, following the same protocol. In view of the results obtained, it was found that both inoffice whitening protocols showed significant whitening after the two whitening sessions and there was no considerable difference observed between the groups regarding the absolute risk and intensity of tooth sensitivity. Thus, it can be concluded that the use of a single application of the gel in 40 minutes is more advantageous, as it does not require a gel update, generating a reduced cost.

\section{Tooth Bleaching and Impact on Quality of Life}

Nascimento et al. [10] evaluated the impact of tooth whitening on people's quality of life. For this, the OHIP-14 questionnaire was applied, containing 14 questions divided into 7 aspects, being assessed functional limitation, physical pain, psychological discomfort, physical disability, psychological disability, social disability and social disability, in 40 patients aged 18 and 30 years, undergoing dental whitening in a dental office with $35 \%$ hydrogen peroxide before and 30 days after the procedure. From the results obtained, it was concluded that of the evaluated aspects, the performance of dental whitening in the office improved only the aspect of psychological discomfort of the patients analyzed due to the favoring of aesthetics, however this issue improved the quality of life of these patients.

\section{Bleaching and Oral Tissue}

Colares et al. [11] evaluated whether bleaching agents with hydrogen peroxide could cause collateral damage to gingival tissue after application in a dental office. For this, samples of gingival fluid were obtained from twenty-two patients. The bleaching procedure was performed with $\mathrm{H}_{2} \mathrm{O}_{2}$ in different concentrations, in a randomized, double-blind clinical trial and with the split mouth study model. Samples of crevicular gingival fluid (GCF) were collected for analysis before, 7 and 21 days after bleaching treatment. From the results obtained from the analyzes, it was noted that the GCF samples (gingival liquid) have higher neutrophil content and lower concentrations of NO-x after bleaching with $\mathrm{H} 2 \mathrm{O} 2$ and after the second bleaching session, the concentrations of GCF $\mathrm{NO}-\mathrm{x}$ were similar to those detected in the baseline. In addition, the MPO content was increased (22-fold increase) in the GCF samples from patients who underwent bleaching with $\mathrm{H}_{2} \mathrm{O}_{2}$ compared to the initial measurements. Proteomic analysis of GCF samples showed that tooth whitening causes changes in the abundance of proteins associated not only with $\mathrm{NO}$ and $\mathrm{H}_{2} \mathrm{O}_{2}$ synthesis, but also with neutrophil recruitment. Proteins associated with NO synthesis and oxidative stress increased after bleaching, regardless of $\mathrm{H}_{2} \mathrm{O}_{2}$ concentration. Thus, it can be concluded that, usually, products based on $\mathrm{H}_{2} \mathrm{O}_{2}$ used in the dental office promote inflammation, regardless of their concentration. However, bleaching gels with lower concentrations can result in less tissue damage.

\section{Discussion}

The first desire in most patients looking to improve the aesthetics of their teeth is to make them whiter, which may reflect in improving the quality of life [10]. Teeth bleaching techniques have evolved over the past few years in order to keep up with the changing profile of the patient seeking this type of treatment. More and more new techniques and new products are emerging in order to improve the efficiency of bleaching and reduce any harmful effects that may occur as a result of this procedure. Tooth bleaching is a technique that is safe, efficient and has no harmful effects on dental enamel when an adequate and individualized protocol is performed for each patient [18]. To perform tooth whitening, there are three modalities: home tooth whitening, office tooth whitening and the combination of both techniques (home and office). The 
most used bleaching agent is hydrogen peroxide, which has its concentration varied depending on the modality to which it will be used, and for the home modality, lower concentrations of peroxide (approximately, 6 to $7 \%$ ) are available to the patient oriented to perform the use of bleaching trays with small amounts of bleaching gel. For the practice modality, hydrogen peroxide varies around $35 \%$. The second type of bleaching agent is carbamide peroxide. This carbamide peroxide gel, when in contact with saliva, decomposes into hydrogen peroxide and urea, so it is also effective in tooth whitening. It can be found in lower concentrations that vary around 10 to $20 \%$ with application in the bleaching tray for home use, or in higher concentrations for use in the office.

In addition to the office dental whitening modality, there is the inclusion of a light source in order to activate the whitening gel and accelerate the tooth whitening process [8]. This light source can be halogen light, LED light (light emitting diode) or Laser (acronym for light amplification by stimulated emission of radiation, that is, amplification of light by stimulated emission of radiation). Regarding the use of a light source in the treatment of tooth whitening, there are still many controversies.

Some manufacturers of whitening gels choose to make the use of light necessary for the activation of the oxidation reaction (fundamental for the whitening process), however, they are often the same manufacturers that commercialize the light source, which makes the indication this technique (light whitening) unreliable. The most recent studies point out that the use of light, be it halogen, LED or Laser, as a process accelerator does not influence the effectiveness of the result when compared to the same procedure without the use of light $[1,2]$. Teeth whitening, despite being recognized as a minimally invasive technique, when performed under the necessary recommendations, can cause structural and morphological changes in the pulp, dentin and enamel [15]. These changes may be greater depending on the technique and the product used.

The success of tooth whitening is due to the diffusion of hydrogen peroxide into the tooth, in an oxidation reaction, which breaks large molecules of chromophores into smaller ones. This reaction increases dental permeability. Ayres et al. [12] evaluated the increase in this permeability due to whitening by comparing four high-concentration gels for use in the dental office. In view of the studies pointed out in this review and the limitations presented, it can be concluded that despite the whitening of the office alone, it presents a satisfactory result in less time (immediate), [7] the association of techniques must be well regarded in order to minimize possible damage to dental structures. Thus, the professional is advised to know the product he is going to use, follow the manufacturer's recommendations for application and patient orientation, respect the soft tissue protection technique, use products that minimize sensitivity, such as gels that have in its composition calcium, since the absence of desensitizers increases tooth permeability and the chances of sensitivity and permanent damage [13].

\section{Clinical Significance}

The literature reports that tooth whitening is a technique that is safe, efficient and has no harmful effects on dental enamel when an appropriate and individualized protocol is performed for each patient.

\section{Conclusion}

In view of the evaluated aspects and their limitations, after reviewing the literature on dental bleaching in the office, it can be concluded that the association of the office and home techniques can minimize possible damage to the dental structures; the professional, to obtain favorable results in the use of the office whitening technique, must know the product he will use, follow the manufacturer's recommendations for the application and guidance to the patient, respect the soft tissue protection technique, use products that minimize the sensitivity; in addition to individualizing each treatment. It is suggested the use of gels with moderate concentrations, of neutral or alkaline $\mathrm{pH}$, following the recommendations of time and application to not expose the enamel to a deeper aggression.

\section{Acknowledgement}

All contributing authors have significantly contributed to the research and writing of the manuscript and approved the content before submission.

\section{Conflict of Interest}

Nil.

\section{References}

1. Maia ACL, Catão MHCV (2010) Claremont Dental Laser (470 nm) e Led com Peróxido de Hidrogênio. Rev bras ci Saúde 14(1): 99-108.

2. Nunes MF, Masotti AS, Rolla JN, Soares CG, Conceição EN (2009) Avaliação clínica do efeito de duas técnicas de clareamento dental em consultório utilizando peróxido de hidrogênio. Rev Fac Odontol Porto Alegre 50(2): 8-11.

3. Karaarslan ES, Özmen ZC, Aytac F, Bicakci AA, Buldur M, et al. (2019) Evaluation of biochemical changes in dental tissues after different office bleaching methods. Human and Experimental Toxicology 38(4): 389397.

4. Penha ES, Pinto WT, dos Santos RL, Guênes GMT, de Medeiros LADM, et al. (2015) Avaliação de diferentes sistemas de clareamento dental de consultório. RFO Passo Fundo 20(3): 281-286.

5. Rodrigues FT, Serro AP, Polido M, Ramalho A, Figueiredo-Pina CG (2017) Effect of bleaching teeth with hydrogen peroxide on the morphology, hydrophilicity, and mechanical and tribological properties of the enamel. Wear 374: 21-28.

6. Guth RC, de Castro Filho AA, de Castro SL, Gagliardi RM (2012) Clareamento dental de consultório em dentes vitais com Whiteness HP Blue 20\% e Whiteness HP Maxx 35\% - Relato de caso clínico. Revista Dentística on line 11(23): 33-37. 
7. Rezende M, Siqueira SH, Kossatz S (2014) Clareamento dental - efeito da técnica sobre a sensibilidade dental e efetividade. Rev Assoc Paul Cir Dent 68(3): 208-212.

8. Maran BM, Burey A, Matos TP, Loguercio AD, Reis A (2018) In-office dental bleaching with light vs. without light: a systematic review and meta-analysis. Journal of Dentistry 70: 1-13.

9. Lima SNL, Ribeiro IS, Grisotto MA, Fernandes ES, Hass V, et al. (2018) Evaluation of several clinical parameters after bleaching with hydrogen peroxide at different concentrations: A randomized clinical trial. Journal of Dentistry 68: 91-97.

10. Nascimento LSB, Lima SNL, Ferreira MC, Malheiros AS, Tavarez RRJ (2018) Avaliação do impacto do clareamento dental na qualidade de vida de pacientes adultos. Journal Health NPEPS 3(2): 392-401.

11. Colares VLP, Lima SNL, Sousa NCF, Araújo MC, Pereira DMS, et al. (2019) Hydrogen peroxide-based products alter inflammatory and tissue damage-related proteins in the gingival crevicular fluid of healthy volunteers: a randomized trial. Scientific reports 9(1): 1-11.

12. Ayres APA, Berger SB, Carvalho AO, Giannini M (2016) Efeito do peróxido de hidrogênio na permeabilidade dental. Rev bras odontol 73(2): 96100.

13. Gonçalves MLL, Tavares ACS, Da Mota ACC, Penna LAP, Deana AM, et al. (2017) In-office tooth bleaching for adolescents using hydrogen peroxide-based gels: clinical trial. Brazilian Dental Journal 28(6): 720725.

14. Loretto SC, Libdy MR, Ribeiro FSR, Braga EMF, Carneiro KGK, et al. (2013) Influence of whitening gel on pulp chamber temperature rise by in-office bleaching technique. Rev Odontol UNESP 42(6): 432-438.

15. Sun L, Liang S, Sa Y, Ma X, Jiang T, et al. (2011) Surface alteration of human tooth enamel subjected to acidic and neutral $30 \%$ hydrogen peroxide. Journal of dentistry 39(10): 686-692.

16. Pinto AVD, Bridi EC, Amaral FLB, França FMG, Turssi CP, et al. (2017) Enamel mineral content changes after bleaching with high and low hydrogen peroxide concentrations: colorimetric spectrophotometry and total reflection X-ray fluorescence analyses. Operative Dentistry 42(3): 308-318.

\section{ISSN: 2574-1241}

\section{DOI: 10.26717/BJSTR.2021.35.005694}

Luis Eduardo Genaro. Biomed J Sci \& Tech Res

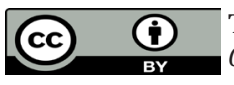

This work is licensed under Creative Commons Attribution 4.0 License

Submission Link: https://biomedres.us/submit-manuscript.php
17. Moreira RF, Santos FP, Santos EA, dos Santos R. S, dos Anjos MJ, (2017) Analysis of the chemical modification of dental enamel submitted to $35 \%$ hydrogen peroxide "In-Office" whitening, with or without calcium. International Journal of Dentistry 2017.

18. De Rosa A, Di Stasio D, Lauritano D, Santoro R, Marotta A, et al. (2019) Non-invasive analysis of bleaching effect of hydrogen peroxide on enamel by reflectance confocal microscopy (RCM): study of series of cases. Odontology 107(3): 285-290.

19. Acuña ED, Parreiras SO, Favoreto MW, Cruz GP, Gomes A, et al. (2019) In-office bleaching with a commercial $40 \%$ hydrogen peroxide gel modified to have different pHs: Color change, surface morphology, and penetration of hydrogen peroxide into the pulp chamber. J Esthet Restor Dent :1-6.

20. Mondelli RFL, Azevedo JFDG, Francisconi AC, Almeida CMD, Ishikiriama SK (2012) Comparative clinical study of the effectiveness of different dental bleaching methods - two-year follow-up. J Appl Oral Sci 20(4): 435-443.

21. Grazioli G, Valente LL, Isolan CP, Pinheiro HA, Duarte CG, et al. (2018) Bleaching and enamel surface interactions resulting from the use of highly concentrated bleaching gels. Archives of Oral Biology 87: 157162.

22. Roderjan DA, Stanislawczuk R, Hebling J, Costa CAS, Reis, A, et al. (2015) Response of human pulps to different in-office bleaching techniques: preliminary findings. Braz Dent J 26(3): 242-248.

23. Izquierdo-Barba I, Torres-Rodríguez C, Matesanz E, Vallet-Regí M (2015) New approach to determine the morphological and structural changes in the enamel as consequence of dental bleaching. Materials Letters 141: 302-306.

24. Martins IEB, Onofre S, Franco N, Martins LM, Montenegro A, et al. (2018) Effectiveness of in-office hydrogen peroxide with two different protocols: a two-center randomized clinical trial. Operative Dentistry 43(4): 353-361.

25. Menezes RP, Silva PD, Leal PC, Faria-e-Silva AL (2018) Impact of 35\% hydrogen peroxide on color and translucency changes in enamel and dentin. Braz Dent J 29(1): 88-92.

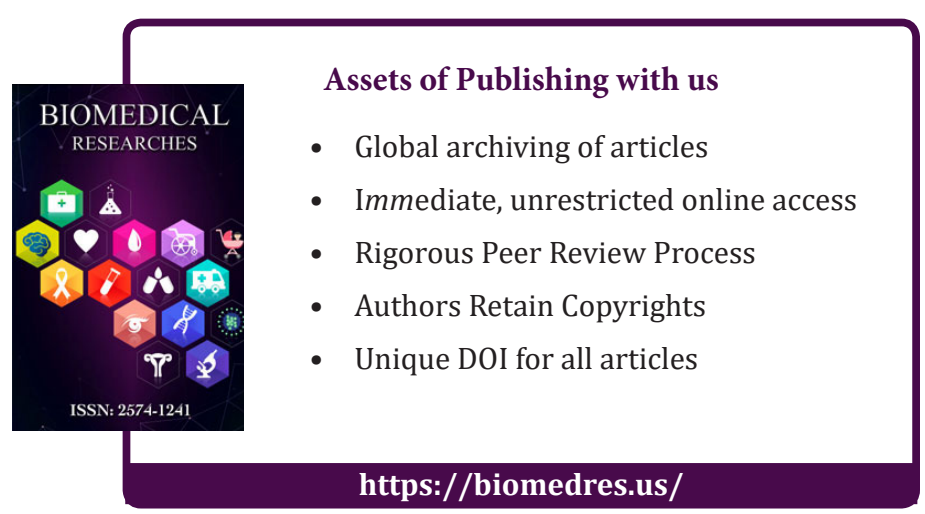

